\title{
Two-Part Marginal Cost Pricing Equilibria: Existence and Efficiency*
}

\author{
Donald J. Brown \\ Stanford University, Stanford, California 94305 \\ WALTer P. Heller \\ University of California, San Diego, San Diego, California 92093 \\ AND \\ Ross M. STARr \\ University of California, San Diego, San Diego, California 92093
}

Received November 26, 1990; revised September 19, 1991

\begin{abstract}
Two-part tariffs are explored in a general equilibrium model with increasing returns to scale. Two-part marginal cost pricing equilibria are not generally Paretoefficient. The Second Fundamental Theorem of Welfare Economics may also fail. We introduce a notion of consumer surplus as the willingness to pay for access to the increasing returns good. The individuals's hookup charge is set to a fixed fraction of his consumer surplus. If aggregate consumer surplus exceeds the losses of the regulated monopoly, then exact two-part marginal cost pricing equilibria exist. Further, for efficient allocations having positive net surplus, the Second Fundamental Theorem of Welfare Economics holds. Journal of Economic Literature Classification Numbers: C62, D42, D61. 1992 Academic Press, Inc
\end{abstract}

\section{INTRODUCTION}

We explore the existence and efficiency of equilibrium two-part tariff pricing in a general equilibrium model with increasing returns to scale. Specifically, consider a limited liability private ownership economy with a regulated natural monopoly that operates under increasing returns. Competitive firms with nonincreasing returns necessarily price at marginal cost. The natural monopoly uses a two-part tariff pricing rule: the losses incurred by pricing at marginal cost are recovered by a hookup charge for access to any purchases of the monopoly good.

* The first author gratefully acknowledges the support of the National Science Foundation. The second author wishes to thank the Graduate School of Business at Stanford University for providing a stimulating environment during his visit. We also thank Duncan Foley, Andreu Mas-Colell, Raylynn Oliver, Kwan Koo Yun, and the JET referee for helpful comments. 
In particular, the first part of the pricing rule is a fixed charge that is imposed on any buyer wishing to purchase any positive amount of the increasing returns good. This is the hookup charge. The hookup charge may be uniform across buyers or it may vary across buyers. The second part of the pricing rule is the variable charge: it consists of a constant per unit charge equal to the marginal cost of production. The constant is the same for all buyers. The natural monopoly is regulated as follows:

(a) it must price each unit sold at the marginal cost of the last unit it produces;

(b) it may set hookup charges so as cover any losses incurred from marginal cost pricing.

We develop a concept of the consumer's willingness to pay (or surplus) for an increasing returns good. Given the budget constraint, the surplus is the compensating variation of consuming the good compared to the best that could be attained if the good were not available. Our regulated monopoly pricing rule is that the buyer's hookup charge is set to be a fraction of the buyer's surplus from the increasing returns food. This construction of a proportional hookup rule is unique to this paper (see expression (*) below).

There is substantial literature on the existence and Pareto-efficiency of marginal cost pricing equilibria in economies with increasing returns. See, for example, Ruggles [18] for a survey of the earlier partial equilibrium literature. The recent general equilibrium literature is ably surveyed in the introduction by Cornet [9] to the Journal of Mathematical Economics Symposium on increasing returns [19]. This Symposium also presents much of the current research on the subject. In this literature, the losses incurred by increasing returns firm's pricing at marginal cost are directly subsidized by lump sum taxes.

However, subsidizing firms from taxes of any kind is rarely observed in Western market economies. ${ }^{1}$ Regulated natural monopolies are usually required to recover losses in the marketplace. Average cost pricing is frequently used, though the resulting allocation has no hope of being Pareto-efficient. For this reason, two-parts tariffs have long been discussed by economists as a means of reconciling efficiency without using subsidies, albeit in partial equilibrium. By contrast with subsidies to firms, two-part tariffs are already in widespread use in Western market economies.

We consider an Arrow-Debreu model of a private ownership economy with limited liability shareholding, ${ }^{2}$ where one firm is a natural monopoly.

\footnotetext{
${ }^{3}$ Electricite de France is perhaps the main exception.

${ }^{2}$ In much of the general equilibrium marginal cost pricing literature, shares in the firm carry unlimited liability. In effect, shares constitute the lump sum taxes necessary to cover the losses of the natural monopoly. Therefore, marginal cost pricing equilibria need not be individually rational.
} 
Its production set is non-convex. This firm is regulated and is required to price at marginal cost. Furthermore, the natural monopoly may recover all the losses it incurs by charging households a fixed charge for the right to consume its products. For the sake of simplicity, we may assume the natural monopoly produces a single output. Finally, we suppose that other firms may purchase the natural monopoly's output, but they are not charged a hookup; this is discussed below. All other firms are profitmaximizing with convex technologies. The natural monopoly's hookup charge also makes the consumers' budget sets nonconvex.

The double nonconvexity of the natural monopoly production set and the budget set complicates the proof of existence of equilibrium. However, exact equilibria do exist. These two-part tariff equilibrium are called Two-Part Marginal Cost Pricing Equilibria (TPMCPE).

Let us clarify the relationship between two-part tariff equilibria and marginal cost pricing equilibria. The two-part tariff equilibria of this paper are individually rational by our proportional hookup rule (see expression (*) below). It is easy to see that many marginal cost pricing equilibria make consumers worse off than doing without the increasing returns good. Therefore, such equilibria cannot be two-part tariff equilibria. Conversely, every two-part tariff equilibrium is a marginal cost pricing equilibrium: the variable charge is set at marginal cost, while the hookup charge can be construed as a lump sum tax. Two-part tariff equilibria constitute a proper subset of the set of marginal cost pricing equilibria. ${ }^{3}$

It is useful to distinguish two types of allocative inefficiencies that may occur in marginal cost pricing equilibrium:

Type 1. The increasing returns good is subsidized and produced in positive quantities when efficiency requires that it not be produced at all. This will arise if the natural monopoly's fixed costs are large compared to the value of consumption benefits. In this case a trivial equilibrium (i.e., zero natural monopoly output) is the only efficient solution (see Coase $[8]$ ).

Type 2. There is more than one non-trivial marginal cost pricing equilibrium and some are Pareto inferior to others.

A benefit-cost criterion sufficient to avoid a type 1 failure is simply that the losses of the firm be less than the aggregate payment households would willingly make for access to the increasing returns good. This criterion is precisely the market test implicit in a two-part tariff equilibrium: the sum of household acess payments equals the firm's losses. Under our proportional hookup rule, hookups cannot exceed consumer surplus, so

${ }^{3}$ This is to even when there are identical consumers; see the example in Section V. 


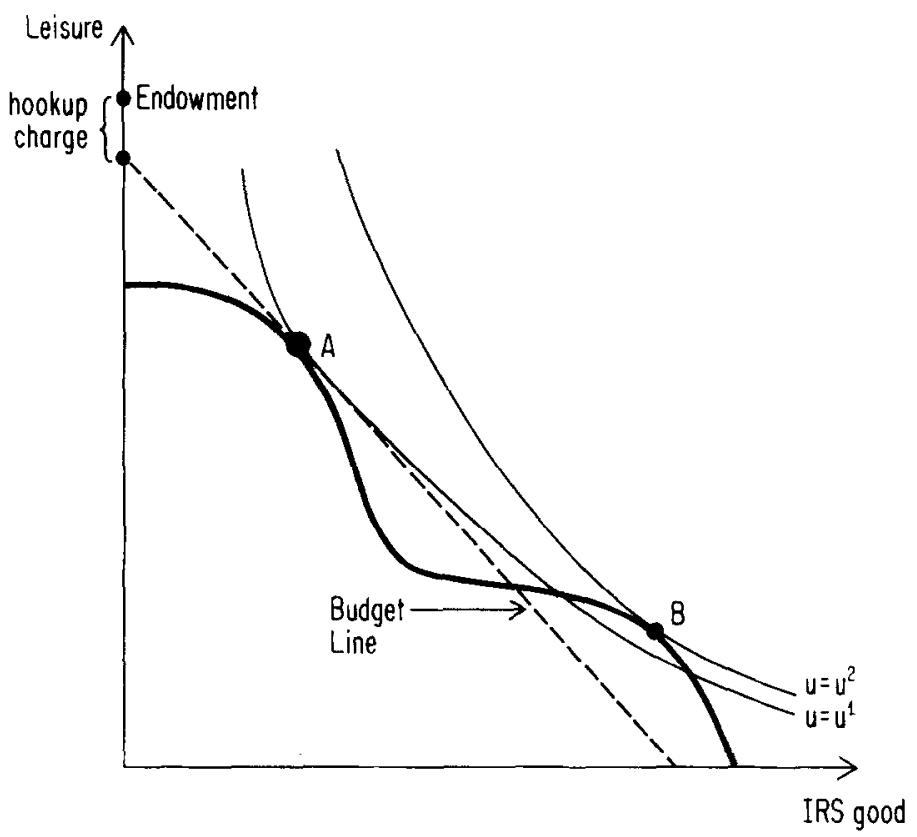

Fig. 1. Failure of the first fundamental theorem.

type 1 inefficiencies cannot occur with our two-part tariffs. However, the inefficiencies associated with type 2 remain, and will be discussed below.

As already mentioned, we establish a condition that is sufficient for the existence of non-trivial exact two-part tariff equilibria. The standard assumptions from the marginal cost pricing equilibrium existence literature (e.g., Bonnisseau and Cornet [3]) are also used here. The additional assumption needed for our analysis of two-part tariffs is the benefit-cost criterion of the previous paragraph. Namely, at the marginal cost prices associated with any candidate equilibrium, the aggregate willingness to pay for the natural monopoly's output exceeds the losses incurred by marginal cost pricing of that output. When this condition holds, two-part marginal cost pricing equilibria exist. Further, these equilibria involve positive production of the increasing returns monopoly good under our proportional hookup rule.

In a partial equilibrium setting, it intuitive that two-part tariff equilibria should reproduce the efficiency properties of competitive equilibrium. ${ }^{4}$ The intuition seems compelling, inasmuch as two-part tariff equilibria fulfill first order conditions and are individually rational. This view is simply mistaken

${ }^{4}$ For a discussion of this view, see Lewis [14], Coase [8], and Oi [16]. 
in general equilibrium. These tests are local; in the nonconvex setting, a local optimum need not be globally optimal. We show on the contrary that two-part marginal cost pricing equilibria are not generally Pareto-efficient. ${ }^{5}$

Inefficiency of ordinary marginal cost pricing equilibria is well-known in the general equilibrium pricing literature (cf., Guesnerie [11], Brown and Heal [6], and Beato and Mas-Colell [2]). However, there are fewer twopart tariff equilibria, so one might have hoped for efficiency of two-part tariff equilibria. That this is false is clear from the example given by Fig. 1; the two goods are leisure and an increasing returns to scale (IRS) good. Point $\mathrm{A}$ is a two-part marginal cost pricing equilibrium that is dominated by $B$. Note, however, that point $B$ can be supported as a Pareto-efficient two-part marginal cost pricing equilibrium. ${ }^{6}$

Since the natural monopoly is regulated in our approach, these results have an important consequence for public policy: the induction of two-part tariff pricing need not result in an efficient outcome. Moreover, some Pareto efficient allocations cannot be supported as two-part tariff equilibria (a result first demonstrated by Quinzii [17]). However, if there is sufficient willingness to pay, then an efficient allocation can be supported by our two-part pricing rule (e.g., point B in Fig. 1).

\section{THE MODEL}

Good zero is the increasing returns good produced by the natural monopoly. Correspondingly, firm zero is the natural monopoly. The Latin letters $x$ and $y$ will denote consumption and production activities, respectively. The indices $h, f$, and $k$ will be used for households, firms, and commodities, respectively. The total number of households, firms, and

\footnotetext{
${ }^{5}$ After many of the results of this paper were completed, an interesting paper by Vohra [20] came to our attention. He examines similar questions. His positive existence and efficiency results are limited primarily to the case of two goods with a pure set-up cost monopoly. He shows, among other things, that inefficient two-part marginal cost pricing equilibria are possible even with nonuniform fixed charges.

${ }^{6} \mathrm{~A}$ simple analytical example follows. Let there be two firms 0,1 with production technologies $Y^{0}, Y^{1}$ characterized by the production functions

$$
\begin{aligned}
f^{0}\left(L^{0}\right) & =\left\{\begin{array}{lll}
L^{0} & \text { for } & L^{0} \leqslant 1.2 \\
8\left(L^{0}-1.2\right)+1.2 & \text { for } & L^{0}>1.2 .
\end{array}\right. \\
f^{1}\left(L^{1}\right) & =2 L^{1} .
\end{aligned}
$$

There is a single household with labor endowment $\bar{L}=2.4$, and the economy's resource constraint is $L^{0}+L^{1} \leqslant \bar{L}$. Let household preferences be characterized by $u\left(x^{0}, x^{1}\right)=$ $\min \left[x^{1}+4 x^{0}, 6 / 7\left(3 x^{1}+x^{0}\right)\right]$. Let the wage rate be $w=2$. Then $(1.2,2.4)=\left(y^{0 *}, y^{1 *}\right)$ is a feasible allocation with $L^{0}=1.2, L^{1}=1.2 .\left(p^{0}, p^{1}\right)=(1,1)$ fulfills marginal cost price. Firm $0^{\text {'s }}$ loss is 1.2 , so a hookup charge of 1.2 results in $(1.2,2.4)$ as a TPMCPE allocation. But $\left(y^{0}, y^{1}\right)=(10.8,0)$ is preferable and attainable.
} 
commodities is $H, F+1$, and $l+1$, respectively. Superscripts on activities $x$ and $y$ will denote the agent performing them; subscripts will denote the commodity. Thus, $x_{k}^{h}$ is the consumption of good $k$ by household $h$, while $y_{k}^{f}$ is the production or input use level of $k$ by firm $f$ (inputs are denoted by negative numbers).

\section{A. Firms}

There is single natural monopoly producing with an increasing returns technology, $Y^{0}$. A technologically feasible production plan for firm 0 is $y^{0} \in Y^{0}$ with $y^{0}=\left(y_{0}^{0}, y_{1}^{0}, y^{0}, \ldots, y_{l}^{0}\right)$. Let the purchases of good 0 by households be given by the vector $x_{0}=\left(x_{0}^{1}, x_{0}^{2}, \ldots, x_{0}^{H}\right)$; the purchases of good 0 by other firms is represented by $y_{0}=\left(y_{0}^{1}, y_{0}^{2}, \ldots, y_{0}^{F}\right)$.

The natural monopoly is a regulated firm, subject to a two-part marginal cost pricing rule with a zero profit constraint. Thus, the pricing rule is a function of the particular plan and the prevailing prices of other goods. Let $q^{h}$ be the hookup charge to household $h$ it buys a positive amount, $x_{0}^{h}>0$. After the initial fixed charge, there is a uniform variable charge $p_{0}$ per unit. The firm takes as given the vectors of output prices $p=\left(p_{0}, p_{1}, \ldots, p_{l}\right)$ and hookup charges for households $q=\left(q^{1}, q^{2}, \ldots, q^{H}\right)$. Below (in $\left.(*)\right)$, we construct a pricing rule such that the hookup charge is zero on any household that chooses to consume zero units of the monopoly good. Then profits for firm 0 at plan $y^{0}$ are given by the function

$$
\tilde{\Pi}^{0}\left(y^{0}, p, q\right)=\sum_{h=1}^{H} q^{h}+p \cdot y^{0}, \quad \text { where } \quad q^{h}=0 \quad \text { if } \quad x_{0}^{h}=0 .
$$

All other firms operate with convex technologies, $Y^{f}$ and are purely competitive in all markets. A typical production plan for firm $f(f \geqslant 1)$ is $y^{f} \in Y^{f},(f=1, \ldots, F)$ with $y^{f}=\left(y_{0}^{f}, y_{1}^{f}, y_{2}^{f}, \ldots, y_{l}^{f}\right)$. An explicit assumption is that no firm other than 0 can produce good $0: y_{0}^{f} \leqslant 0$, for $f=1,2, \ldots, F$. For these competitive firms, profits are

$$
\tilde{\Pi}^{f}\left(y^{f}, p\right)=p \cdot y^{f}
$$

for $f=1, \ldots, F$. Maximized profits will be denoted $\Pi^{f}(p)$.

Let $\partial Y^{f}$ be the boundary of $Y^{f}$. Next, for $y^{f} \in \partial Y^{f}$, define a correspondence $\varphi^{f}\left(y^{f}\right)$ to be the Clarke normal cone to $\partial Y^{f}$ at $y^{f}$, intersected with the simplex. The formal definition of the Clarke normal cone is given in the footnote ${ }^{7}$ for convenience. The motivation is as follows: a conven-

\footnotetext{
${ }^{7}$ Let $Y$ be a closed subset of Euclidean space $\mathbb{R}^{n}$. Consider first the Clarke tangent cone $T_{Y}(y)$ of a point $y \in Y$. It consists of vectors $x$ in $\mathbb{R}^{n}$ such that for all sequences $\left\{y^{v}, t^{n}\right\}$ with $y^{y} \rightarrow y$ and $0<t^{v} \rightarrow 0$, there exists a sequence $x^{v} \rightarrow x$ such that $y^{\prime \prime}+t^{\prime \prime} x^{\prime \prime} \in Y$ for all $v$ large. The Clarke normal cone is polar to this:
}

$$
N_{Y}(y) \equiv\left\{p \in \mathbb{R}^{n} \mid p \cdot x \leqslant 0 \text { for all } x \in T_{Y}(y)\right\} .
$$


tional normal cone to a point on the boundary of a convex production set is simply the set of marginal rates of transformation at that point. The Clarke cone reduces to a conventional normal cone if $Y$ is a convex set (see below).

We now present the assumptions on production sets in Beato and Mas-Colell [2], as articulated by Bonnisseau and Cornet [3]:

(F1) $Y^{f}=K^{f}-R_{+}^{l+1}, \quad$ where $K^{f}$ is compact.

(F2) $K^{f}$ is convex for $f \geqslant 1$.

(F3) The output of firm 0 cannot be produced by any other firm.

(F4) The pricing rule is given by $\varphi^{f}: \partial Y^{f} \rightarrow \Delta$, where $\Delta$ is the unit simplex in $R^{l}$ and $\varphi^{f}\left(y^{f}\right)$ is the Clarke normal cone intersected with the simplex.

$(\mathrm{F} 1)$ and $(\mathrm{F} 4)$ have as a consequence:

(F5) There exists $\bar{r}>0$ such that for all $f, K^{f}$ is contained in the interior of $\{-\bar{r} e\}+R_{+}^{\prime}$, where $e=(1,1, \ldots, 1)$. Moreover, if $y_{k}^{f} \leqslant-\bar{r}$ and $p \in \varphi^{f}\left(y^{f}\right)$ then $p_{k}=0$.

For a proof of the boundary condition in (F5), see Bonnisseau and Cornet [3], Lemma 4.2. The reader should think of the $K^{f}$ as proxies for the attainable production sets of the firms. See Bonnisseau and Cornet [3], Section 3.2, for an exposition of this intuition.

\section{B. Households}

There are $H$ households, where each household is characterized by its consumption set $\left(X^{h}\right)$, endowments $\left(\omega^{h}\right)$, utility functions $\left(U^{h}: X^{h} \rightarrow \mathbb{R}\right)$, and shareholdings in firms $\left(\theta^{h f}\right.$, where $\sum_{h} \theta^{h f}=1$, and $\theta^{h f} \geqslant 0$, for $f=0,1, \ldots, F$.) Note that households own shares in the natural monopoly.

There is a large and growing literature on marginal cost pricing in general equilibrium (e.g., Beato and Mas-Colell [2], Bonnisseau and Cornet [3], Brown, Heal, Khan, and Vohra [7], and Kamiya [13]). Unlike this recent literature, we assume limited liability for the shareholders of the profits of the natural monopoly, $\widetilde{\Pi}^{0}\left(y^{0}, p, q\right)$. Thus, total household dividend distributions from the natural monopoly are $\Pi^{0}\left(p, q, y^{0}\right) \equiv$ $\max \left(0, \tilde{\Pi}^{0}\left(y^{0}, p, q\right)\right)$. For $f \geqslant 1$, the profits $\Pi^{f}$ in (3) are all functions of $p$ alone. For $f=0, \Pi^{0}$ depends on $p, q$, and $y^{0}$. The dividend total for household $h$ is

$$
\pi^{h}\left(p, q, y^{0}\right) \equiv \theta^{h 0} \Pi^{0}\left(p, q, y^{0}\right)+\sum_{f=1}^{\digamma} \theta^{h f} \Pi^{f}(p), \quad \text { for all } h .
$$

Income is $p \cdot \omega^{h}+\pi^{h}\left(p, q, y^{0}\right)$. Expenditures are $q^{h}+p \cdot x^{h}$ if $x_{0}^{h}>0$, and $p \cdot x^{h}$ if $x_{0}^{h}=0$. 
The budget correspondence, $B^{h}\left(p, q, y^{0}\right)$, is defined for all $h$ as

$$
B^{h}\left(p, q, y^{0}\right) \equiv\left\{\begin{array}{lll}
x^{h} \in X^{h} & \begin{array}{l}
p \cdot x^{h} \leqslant p \cdot \omega^{h}+\pi^{h}, \\
q^{h}+p \cdot x^{h} \leqslant p \cdot \omega^{h}+\pi^{h},
\end{array} & \text { if } \quad x_{0}^{h} \leqslant 0
\end{array}\right\},
$$

where $\pi^{h} \equiv \pi^{h}\left(p, q, y^{0}\right)$ is given by ( 3$)$.

The standard neoclassical assumptions on households will be used. For $h=1,2, \ldots, H$ :

(H1) $X^{h} \subset \mathbb{R}_{+}^{l+1}$ is nonempty, closed and convex.

(H2) For all $x^{h} \in X^{h},\left(0, x^{\prime h}\right) \in X^{h}$, where for $k \geqslant 1, x_{k}^{\prime h}=x_{k}^{h}$.

(H3) $U^{h}$ is continuous.

(H4) $U^{h}$ is strictly quasi-concave.

We assume in (H2) that purchases of natural monopoly output is never necessary for survival. The compactness of the values of $B^{h}$ is ensured by restricting consumer choices to a compact set containing the attainable consumption set $\hat{X}^{h}$ in its interior. Call the result $\bar{B}^{h}\left(p, q, y^{0}\right)$. The excess demand correspondence $\bar{d}^{h}\left(p, q, y^{0}\right)$ is the set of maximizers of $U^{h}$ subject to being in $\bar{B}^{h}\left(p, q, y^{0}\right)$. Under the assumptions made below we will show that $\bar{d}^{h}$ is upper hemicontinuous, but not in general convex-valued. The latter property comes about because of the non-convexity of $B^{h}$. However, we will also show that $d^{h}$ is convex-valued over the relevant region of its domain, i.e., the prices corresponding to the production equilibria. Because of strict quasi-concavity, $d^{h}$ can be therefore represented as a continuous function on that region.

\section{Two-Part Marginal Cost Pricing Rules}

As already stated, the pricing rule is applied by the regulated natural monopoly firm producing good 0 . The hookup charge can be set individually to extract some fixed proportion of the consumer surplus for each household. At another extreme, it could be some arbitrary amount that is uniform across consumers, and we call this scheme a uniform hookup charge. The difficulty with any particular hookup charge is that some households may be willing to pay the variable charge, while at the same time refuse to buy good 0 . In this case, we will call the hookup charge exclusionary.

Consider a simple case where demand is given by a function $x_{0}=d\left(p_{0}\right)$. The natural monopoly technology can be represented by a single input convex production function $y_{0}=f(L)$. The variable charge is set equal to marginal costs: $p_{0}=1 / f^{\prime}$, where it is supposed that the wage rate is numeraire. Solve these equations to get partial equilibrium values $p_{0}^{*}, L^{*}$. Hookup charges must sum to equal total losses $F^{*} \equiv L^{*}-p_{0}^{*} f\left(L^{*}\right)>0$ (by 
convexity of $f$ ). If $F^{*} / H$ is less than the consumer surplus of representative consumer for good 0, then a Pareto-efficient equilibrium has been found, This is a standard partial equilibrium two-part tariff analysis. On the other hand, if there are heterogeneous consumers, then it is quite possible that $F^{*} / H$ is above the consumer surplus of some household.

It is of course inefficient to exclude such households with a too-high hookup charge. The regulator wishes to impose a pricing rule on the monopoly that simultaneously ensures Pareto-efficiency and nonnegative monopoly profits. With a two-part tariff, this means a variable charge equal to marginal cost and hookup charges that cover losses from priving below average cost, yet are not exclusionary. It is not at all clear that these are compatible goals in a general equilibrium economy with a nonconvex technology for the natural monopoly. In fact, it will be shown that although a nonexclusionary general equilibrium exists, it need not be Pareto-efficient.

In order to avoid the obvious inefficiencies associated with exclusion, we will below define a notion of consumer surplus arising from the monopoly good. The hookup charge to any household will then be some fraction of that household's surplus. At any price vector, the only households who do not choose to buy the monopoly good be facing a hookup charge of zero.

This is how we avoid the discontinuities of demand that arise from nonconvex budget sets. We do not need to rely on concepts and arguments involving approximate equilibria as in Heller and Starr [12]. Any point of discontinuity must involve a jump to zero consumption of the monopoly good, owing to the structure of the constraint set $B^{h}$. But on all occasions when someone would choose to consume zero of the monopoly good, his hookup is 0 , and so his budget constraint is convex at those prices. Therefore, demand is continuous.

\section{Existence of Two-Part Marginal Cost Pricing Equilibria}

The basic idea of our proof rests on the aggregate willingness to pay of households, for the output of the natural monopoly. When aggregate willingness to pay exceeds the natural monopoly's losses, our hookup pricing rule allows each household some positive surplus after paying the hookup. If a household has no desire for the natural monopoly's output, their hookup is zero. This means that no agent is indifferent between demanding a strictly positive amount of the monopoly good and demanding zero amount. Hence demand functions of households will be continuous.

Our proof closely resembles that of Beato and Mas-Colell [2], except that we do not assume differentiable production frontiers and we seek a 
two-part tariff equilibrium. Because of the nondifferentiability, the marginal part of our pricing rule $\varphi^{f}\left(y^{f}\right)$ is defined by the Clarke normal cone. For more on Clarke normal cones, see Bonnisseau and Cornet [3], especially Lemma 4.1. Given our assumptions on $Y^{f}, \varphi^{f}(\cdot)$ is a nonempty convexvalued and upper hemi-continuous correspondence.

We can approximate the marginal part of the pricing rule by functions whose graphs are arbitrarily close to the graph of the marginal cost pricing correspondence. For the approximation result, see von Neumann's Approximation Lemma in Border [5]. We shall denote a given approximation to the marginal cost pricing correspondence of firm $f$ by $g^{f}$, $g^{f}: \partial Y^{f} \rightarrow \Delta$, where $g^{f}$ is a continuous function. For the competitive firms, $g^{f}$ completely specifies their pricing rule.

The pricing rule for the natural monopoly is two-part marginal cost pricing. The variable charge per unit is equal to marginal cost, i.e., if $y^{0} \in Y^{0}$, then $p^{0}=g^{0}\left(y^{0}\right)$. The natural monopoly also charges each household a hookup charge $q^{h}\left(p, y_{0}\right) \geqslant 0$ for the right to consume its output. The hookup charges are then set so that the losses are exactly covered by the hookup charges: $-p \cdot y^{0}=\sum_{n} q^{h}\left(p, y^{0}\right)$. Hookup charges are zero for any household who does not buy the monopoly good. They are also zero for all firms $f$.

With this pricing rule for the natural monopoly, we can drop the dependence on $q$ for $\Pi^{0}\left(y^{0}, p, q\right)=\max \left(0, p \cdot y^{0}\right) \equiv \Pi^{0}\left(y^{0}, p\right)$.

Definition. A production equilibrium is a pair $\left(p,\left(y^{f}\right)\right)$ where, for all $f$, $p \in \varphi^{f}\left(y^{f}\right)$ and $y^{f} \in \partial Y^{f}$; PE will denote the set of all production equilibria.

Next, the attainable sets are compact. Let $\omega=\sum \omega^{h}$. Assumptions (F.1) and (H.1) guarantee that the attainable sets of households, denoted $\hat{X}^{h}$, are compact. This is a consequence of the fact that if $Y \equiv \sum Y^{f}$ then $(Y+\omega) \cap R_{+}^{\prime}$ is compact and the $X^{h} \subset R_{+}^{l}$.

We shall make the following assumptions on PE. For any production equilibrium, there is in aggregate enough income to purchase survival consumption levels for everybody.

(SA) $\quad\left(p,\left(y^{f}\right)\right) \in \mathrm{PE}$ implies $p \cdot\left(\sum_{0}^{F} y^{f}+\omega\right)>\inf p \cdot \sum_{h} \hat{X}^{h}$

Define $r^{h}: \mathrm{PE} \rightarrow \mathbb{R}$ to be income of household $h$,

$$
r^{h}\left(p,\left(y^{f}\right)\right) \equiv p \cdot \omega^{h}+\theta^{h 0} \Pi^{0}\left(y^{0}, p\right)+\sum_{f=1}^{F} \theta^{h f} p \cdot y^{f},
$$

where $\left(p,\left(y^{f}\right)\right) \in \mathrm{PE}$. Assumption (R) below says, in conjunction with (SA), that at any production equilibrium there is enough income for the 
household to survive. Assumptions (R) and (SA) are independent of each other.

$$
\begin{gathered}
\text { (R) }\left(p,\left(y^{f}\right)\right) \in \mathrm{PE} \quad \text { and } \quad p \cdot \sum_{0}^{F} y^{f}+p \cdot \omega>\inf p \cdot \sum_{h} \hat{X}^{h} \text { implies } \\
r^{h}\left(p,\left(y^{f}\right)\right)>\inf p \cdot \hat{X}^{h}
\end{gathered}
$$

Finally, we need to ensure that there is sufficient aggregate consumer surplus from the natural monopoly to cover its losses. If $\left(p,\left(y^{f}\right)\right)$ is a production equilibrium, calculate for each household $h$ the "reservation level of utility." This is the maximum utility level she could achieve, if the natural level of utility." This is the maximum utility level she could achieve, if the natural monopoly good was unavailable. Let $Q$ be a large compact "box" that contains all the $\hat{X}^{h}$ in its interior, and define $\bar{X}^{h} \equiv X^{h} \cap Q$. Then $\bar{X}^{h}$ is convex, compact, and contains $\hat{X}^{h}$.

$$
\bar{U}^{h}\left(p,\left(y^{f}\right)\right) \equiv \max U^{h}\left(x^{h}\right) \text { over } x^{h}
$$

such that

$$
\begin{aligned}
x^{h} & \in \bar{X}^{h} \\
p \cdot x^{h} & \leqslant r^{h}\left(p,\left(y^{f}\right)\right) \\
x_{0}^{h} & =0 .
\end{aligned}
$$

Our major conceptual tool will be that of surplus from the increasing returns monopoly good. This is just the "willingness to pay" or consumer surplus for the monopolist's output at $\left(p,\left(y^{f}\right)\right)$ for household $h$,

$$
s^{h}\left(p,\left(y^{f}\right)\right) \equiv r^{h}\left(p,\left(y^{f}\right)\right)-E^{h}\left(p, \bar{U}^{h}\right),
$$

where $E^{h}$ is the expenditure function, i.e.,

$$
E^{h}\left(p, \bar{U}^{h}\right)=\min \left\{p \cdot x^{h} \mid U^{h}\left(x^{h}\right) \geqslant \bar{U}^{h} \text { and } x^{h} \in \bar{X}^{h}\right\} .
$$

The consumer surplus measure is the compensating variation of adding the monopoly good. Namely, it is the amount at current prices that must be subtracted from current income to reduce utility to what it was when the monopoly good was unavailable. In this respect it is related to Dupuit's notion of benefit arising from the introduction of a public good. Of course, $s^{h}$ is an ordinal concept, i.e., it is independent of the utility representation.

We now assume that there is aggregate positive surplus in the economy's willingness to pay, $\sum s^{h}\left(p,\left(y^{f}\right)\right)$. 
(S) For all production equilibria $\left(p,\left(y^{f}\right)\right)$ :

$$
\sum s^{h}\left(p,\left(y^{f}\right)\right)>-\min \left(p \cdot y^{0}, 0\right) .
$$

Condition (S) says that the natural monopoly's losses are smaller than the economy's desire for its output (at any production equilibrium).

Any equilibrium allocation lies in PE. If this inequality failed to hold at every point in PE, the natural monopoly would always run losses that could not be covered by hookup charges. Thus, the natural monopoly could not be active in any equilibrium.

It is worth noting that condition (S) is true if the following condition hold:

(1) there are a sufficiently large number of replicated consumers who strictly ${ }^{8}$ desire the natural monopoly's good, and

(2) the monopoly's losses $-p \cdot y^{0}$ are bounded above.

Bonnisseau and Cornet [3] show that Condition (2) follows from the assumption (F5) above. Therefore, the right hand side of the inequality in (S) is bounded. As an alternative to Condition (1), there could be a small number of households who intensely desire the increasing returns good.

We now define the proportional hookup rule for the natural monopolist and show that it is continuous on PE. Let

$$
s\left(p,\left(y^{f}\right)\right) \equiv \sum_{h} s^{h}\left(p,\left(y^{f}\right)\right)
$$

and

$$
\tau\left(p,\left(y^{f}\right)\right) \equiv \frac{-\min \left(p \cdot y^{0}, 0\right)}{s\left(p,\left(y^{f}\right)\right)} .
$$

Now, $\tau$ is well defined, for by (S), $s\left(p,\left(y^{f}\right)\right)>0$ on PE.

For $\left(p,\left(y^{f}\right)\right) \in \mathrm{PE}$, define the proportional hookup rule:

$$
q^{h}\left(p,\left(y^{f}\right)\right) \equiv \tau\left(p,\left(y^{f}\right)\right) s^{h}\left(p,\left(y^{f}\right)\right) .
$$

The hookup charge for each household is a fraction of her willingness to pay; hence, if the household has positive surplus, she will choose to pay the hookup. The function $q^{h}$ is continuous on PE since $\tau$ and $s^{h}$ are continuous on PE. Note that $s^{h}=0$ if and only if $q^{h}=0$ and that $\sum q^{h}\left(p,\left({ }^{f}\right)\right)=$ $-\min \left(p \cdot y^{0}, 0\right)$.

${ }^{8}$ In fact, $s^{h}$ must be bounded away from 0. 
DefINITION. A two-part marginal cost pricing equilibrium (or TPMCPE) is a vector of prices, consumption plans, and production plans $\left\langle\left(\bar{p},\left(\bar{x}^{h}\right),\left(\bar{y}^{f}\right)\right\rangle\right.$ such that:

(a) All households $h=1, \ldots, H$ are maximizing $U^{h}\left(x^{h}\right)$ at $\bar{x}^{h}$ subject to their budget constraint (4).

(b) Given $\bar{p}$, firms $1, \ldots, F$ maximize profits at $\bar{y}^{f}$; firm 0 prices at marginal cost, $\bar{p} \in \varphi^{0}\left(\bar{y}^{0}\right)$, and imposes the hookup charge $q^{h}\left(\bar{p},\left(\bar{y}^{f}\right)\right)$ on any household that buys its output. ${ }^{9}$

(c) $\sum \bar{x}^{h} \leqslant \sum \bar{y}^{f}+\omega$.

THEOREM 1. Given assumptions (H1)-(H4), (F1)-(F5), (SA), (R), and (S), there exists a TPMCPE in which the natural monopoly has positive output.

Proof. We first show that PE is nonempty. Here we follow the proof of Beato and Mas-Colell [2] and use their map to construct the relevant fixed point. Let

$$
\bar{Y}^{\prime}=Y^{\prime} \cap\left[\{-\bar{r} e\}+R_{+}^{l}\right]
$$

and

$$
\partial \bar{Y}^{f}=\partial Y^{f} \cap\left[\{-\bar{r} e\}+R_{+}^{l}\right] .
$$

Under our assumptions (F1)-(F4), the sets $\bar{Y}^{f}=Y^{f} \cap\left[\{-\bar{r} e\}+R_{+}^{l}\right]$ and $\partial \bar{Y}^{f}$ are compact. Under $(\mathrm{F} 1)$, we may define $\eta^{f}$ to be a homeomorphism of the simplex $A$ onto $\partial \bar{Y}^{f}$ as follows: for each $z^{f} \in \Delta$, the ray $\left\{-r e+\alpha z^{f} \mid \alpha \geqslant 0\right\}$ intersects $\partial \bar{Y}^{f}$ uniquely at the point $\eta^{f}\left(z^{f}\right)$. This homeomorphism maps the $k$ th component of $z^{r}$ into the $k$ th component of $y^{f}$.

Elements of $\Delta$ will be denoted by $z^{f}$, and let $y^{f} \equiv \eta^{f}\left(z^{f}\right)$.

The pricing rules $g_{n}^{f}\left(y^{f}\right)$ are continuous functions from $\partial \bar{Y}^{f}$ into $\Delta$ such that the graph of $g_{n}^{f}\left(y^{f}\right)$ is within distance $1 / n$ of the graph of $\varphi^{f}\left(y^{f}\right)$. Let $\Delta^{F+2}$ be the $(F+2)$-fold Cartesian product of $\Delta$ and $y^{f} \equiv \eta^{f}\left(z^{f}\right)$. Define $\Psi_{n}: \Delta^{F+2} \rightarrow \Delta^{F+2}$ to be

$$
\begin{aligned}
& \Psi_{n}^{i f}\left(\left(z^{f}\right), p\right) \\
& \equiv \frac{z_{j}^{f}+\max \left\{0, p^{j}-g_{n}^{j f}\left(y^{f}\right)\right\}}{1+\sum_{k=1}^{l} \max \left\{0, p^{k}-g_{n}^{k f}\left(y^{f}\right)\right\}}, \quad \text { for } \quad 0 \leqslant f \leqslant F \text { and } 1 \leqslant j \leqslant l \text {. } \\
& \Psi_{n}^{j F+1}\left(\left(z^{f}\right), p\right)=p^{j}, \quad \text { for } 1 \leqslant j \leqslant l
\end{aligned}
$$

\footnotetext{
${ }^{9}$ Note that we include our method of calculating hookups as part of the equilibrium definition. A more general definition of two-part tariff equilibrium would be to merely require zero monopoly profits after collection of hookups.
} 
For each $n$, the continuous function $\Psi_{n}$ has a fixed-point by Brouwer's Theorem, Denote the fixed-point by $\left\langle\left(\bar{z}_{n}^{f}\right) \bar{p}_{n}\right\rangle$, and define $\bar{\rho}_{n}^{f}=g_{n}^{f}\left(\bar{z}_{n}^{f}\right)$. Then taking convergent subsequences we obtain as limits $\left\langle\left(\bar{z}^{f}\right), \bar{p}\right\rangle$. Letting $\bar{\rho}^{f}=g^{f}(\bar{z})$, we observe that $\bar{\rho}^{f} \in \varphi^{f}\left(\bar{z}^{f}\right)$ since the graphs of the $g_{n}^{f}$ converge uniformly to the graph of $\varphi^{f}$. Rewriting Eq. (8) in terms of the fixed points we obtain:

$\bar{z}_{n}^{i f}=\frac{\bar{z}_{n}^{i f}+\max \left\{0, \bar{p}_{n}^{j}-\bar{\rho}_{n}^{i f}\right\}}{1+\sum_{k=1}^{l} \max \left\{0, \bar{p}_{n}^{k}-\bar{\rho}_{n}^{k f}\right\}}, \quad$ for $0 \leqslant f \leqslant F$ and $1 \leqslant j \leqslant l$.

Taking limits, we derive

$$
\bar{z}^{j f}=\frac{\bar{z}^{i f}+\max \left\{0, \bar{p}^{j f}-\bar{\rho}^{j f}\right\}}{1+\sum_{k=1}^{l} \max \left\{0, \bar{p}^{k}-\bar{\rho}^{k f}\right\}} .
$$

Since $\eta^{f}$ preserves faces, $\bar{z}^{j f}=0$ implies $\bar{y}^{i f} \equiv \eta^{j f}\left(\bar{z}^{f}\right)$ is on the face $\bar{y}^{i f}=-r$. Since $\bar{\rho}^{f} \varphi^{f}\left(\eta^{f}\left(\bar{z}^{f}\right)\right)$, the boundary condition in (F5) holds, and so $\bar{\rho}^{i f}=0$. To complete the proof, suppose for some $\hat{f}$ that $\bar{p} \neq \bar{\rho}^{f}$. Then there exists $\hat{j}$ such that $\hat{p}^{j}>\bar{\rho}^{j \hat{f}}$. Hence for all $z^{i f}>0$, we have $\bar{p}^{\prime}>\bar{\rho}^{i f}$. But the boundary condition if $\bar{z}^{i f}=0$ then $\bar{\rho}^{i f}=0$. Hence $\tilde{p}_{j}=0$. Therefore, in all cases, $\bar{p}^{j} \geqslant \bar{\rho}^{i \hat{f}}$ and $\bar{p}^{j}>\bar{\rho}^{\hat{f}}$, a contradiction since $\bar{p}$ and $\bar{\rho}^{f}$ lie on the simplex A. So, $\bar{\rho}^{f}=\bar{p}$ for all $f$ and $\bar{\rho}^{f} \in \varphi^{f}\left(\bar{z}^{f}\right)$. That is, $\mathrm{PE} \neq \varnothing$.

We now show that the aggregate demand function is continuous on PE. Let

$$
\bar{B}^{h}\left(p,\left(y^{f}\right)\right)=\left\{x^{h} \in \bar{X}^{h} \mid q^{h}\left(p,\left(y^{f}\right)\right)+p \cdot x^{h} \leqslant r^{h}\left(p,\left(y^{f}\right)\right)\right\}
$$

for $\left(p,\left(y^{f}\right)\right) \in \mathrm{PE} . \bar{B}^{h}$ is a convex-valued correspondence and compactvalued. We claim that $\bar{B}^{h}\left(p,\left(y^{f}\right)\right)$ is a continuous correspondence on PE. For, recall that $r^{h}(\cdot)$ and $q^{h}(\cdot)$ are continuous. By Assumption (R) $r^{h}(\cdot)>\inf p \cdot \hat{X}^{h}$ on PE. If $q^{h}\left(p,\left(y^{f}\right)\right)=0$, then the standard argument applies (cf. Debreu [10], p. 63). Otherwise $0<q^{h}\left(p,\left(y^{f}\right)\right)<s^{h}\left(p,\left(y^{f}\right)\right)$. Recall that $\bar{U}^{h}\left(p,\left(y^{f}\right)\right)$ is defined in (5) as the reservation level of utility. Also let $\bar{x}^{h}$ be the minimizer in the expenditure function problem (7). Then $0<s^{h}\left(p,\left(y^{f}\right)\right)=r^{h}\left(p,\left(y^{f}\right)\right)-E\left(p \cdot \bar{u}^{h}\left(p,\left(y^{f}\right)\right)\right)$ implies that:

$$
\begin{aligned}
q^{h}(p & \left.\left(y^{f}\right)\right)+p \cdot \tilde{x}^{h} \\
& <s^{h}\left(p,\left(y^{f}\right)\right)+p \cdot \tilde{x}^{h} \\
& =s^{h}\left(p,\left(y^{f}\right)\right)+E\left(p, \bar{U}^{h}\left(p,\left(y^{f}\right)\right)\right) \\
& =r^{h}\left(p,\left(y^{f}\right)\right) .
\end{aligned}
$$

That is, $q^{h}\left(p,\left(y^{f}\right)\right)+p \cdot \bar{x}^{h}<r^{h}\left(p,\left(y^{f}\right)\right)$. Hence $\bar{x}^{h}$ is in the interior of $\bar{B}^{h}\left(p,\left(y^{f}\right)\right)$, and again the standard argument of Debreu applies. 
Now define the demand correspondences

$$
d^{h}\left(p,\left(y^{f}\right)\right)=\operatorname{argmax}\left\{U^{h}\left(x^{h}\right) \mid x^{h} \in \bar{B}^{h}\left(p,\left(y^{f}\right)\right)\right\} .
$$

By the Berge Maximum Theorem, the demand correspondences $d^{h}(\cdot)$ are upper hemi-continuous. But by strict quasi-concavity of $U^{h}$ and convexity of $\bar{B}^{h}, d^{h}(\cdot)$ is a function and therefore is a continuous function. Let $d\left(p,\left(y^{f}\right)\right)=\sum_{h} d^{h}\left(p,\left(y^{f}\right)\right)$; then $d(\cdot)$ is continuous on PE. But PE is a closed subset of $\Delta \times \prod_{f=0}^{F} \partial Y^{f}$. By the Tietze Extension Theorem, $d$ has a continuous extension $\partial(\cdot)$ to all of $\Delta \times \prod_{f=0}^{r} \partial Y^{\prime}$.

We are now ready to complete the proof of the existence of a TPMCPE. Following Beato and Mas-Colell [2] we construct their map. Again, we implicitly use an approximation argument, but the details are much the same as in the existence proof for production equilibria. Define $T: \Delta^{F+2} \rightarrow \Delta^{F+2}$ as follows:

For $0 \leqslant f \leqslant F$ and $0 \leqslant j \leqslant l$, let

$$
T^{j f}(z, p) \equiv \frac{z_{j}^{f}+\max \left\{0, p_{j}-g_{j}^{f}\left(\eta^{f}\left(z^{f}\right)\right)\right\}}{1+\sum_{k=0}^{l} \max \left\{0, p_{k}-g_{k}^{f}\left(\eta^{f}\left(z^{f}\right)\right)\right\}} .
$$

For $f=F+1$ and $0 \leqslant j \leqslant l$, let

$$
T^{j F+1}(z, p) \equiv \frac{p_{j}+\max \left\{0, \sum_{h=1}^{H} \tilde{d}_{j}^{h}(p, \eta(z))-\sum_{f=0}^{F} \eta_{j}^{f}\left(z^{f}\right)-\omega_{j}\right\}}{1+\sum_{k} \max \left\{0, \sum_{h} \tilde{d}_{k}^{h}(p, \eta(z))-\sum_{f} \eta_{k}^{f}\left(z^{f}\right)-\omega_{k}\right\}},
$$

where $\omega \equiv \sum \omega^{h}$.

The continuous map, $T$, has a fixed point $(z, p)$ by Brouwer's Theorem. Let $y^{f}=\eta^{f}(z)$ and consider $\tilde{d}^{h}\left(p,\left(y^{f}\right)\right)$. Then, by the argument in Beato and Mas-Colell [2], $\sum_{h} \partial^{h}\left(p,\left(y^{f}\right)\right) \leqslant \sum_{f} y^{f}+\omega$ (with $p_{k}=0$ if strict inequality). Moreover, by Eq. $(11),\left(p,\left(y^{f}\right)\right)$ is a production equilibrium. Hence, $\widetilde{d}=d$, the aggregate demand. At any production equilibrium there is a positive demand for the monopoly good, see Assumption (S). Therefore, $x_{0}>0$. Since $\omega_{0}=0$, we see that $y_{0}>0$. Also, by construction, at every production equilibrium the sum of the hookups covers the losses of the natural monopoly.

The final step is to show that when households are faced with the hookups $q^{h}(\cdot)>0$, they will not choose to disconnect. There are two cases:

Case 1. If household $h$ has positive surplus $s^{h}\left(p,\left(y^{f}\right)\right)>0$, then it will strictly prefer consuming a bundle with the monopoly good to any other affordable bundle without it. The reason is that (S) guarantees that $\tau\left(p,\left(y^{f}\right)\right)<1$. Thus, when $s^{h}(\cdot)>0, s^{h}(\cdot)-q^{h}(\cdot)>0$. Let $s^{h}<d^{h}\left(p,\left(y^{f}\right)\right)$. Then

$$
p \cdot x^{h}-E\left(p, \bar{U}^{h}\right)=r^{h}(\cdot)-q^{h}(\cdot)-E\left(p, \bar{U}^{h}\right)=s^{h}(\cdot)-q^{h}(\cdot)>0 .
$$


Hence $\bar{U}^{h}\left(x^{h}\right)>\bar{U}^{h}\left(p,\left(y^{f}\right)\right)$. But $\bar{U}^{h}\left(p,\left(y^{f}\right)\right) \geqslant \bar{U}^{h}\left(x^{h}\right)$ for any $x^{h} \in \bar{B}^{h}(\cdot)$ subject to $x_{0}^{n}=0$, and no hookup fee. Hence the constraint $p \cdot x^{h} \leqslant r^{h}$ for $x_{0}^{h}=0$ is not binding.

Case 2. If $s^{h}(\cdot)=0$, then $q^{h}(\cdot)=0$. Hence there are no hookups and we are done.

The final step is to show that $x^{h}$ is optimal not only in $\bar{X}^{h}$ but in all of $X^{h}$. This part of the argument is standard, see Debreu [10], page 57, equation (6).

The proportional hookup rule used in the proof of Theorem 1 is new. All of our constructed equilibria have positive output of the increasing returns good under our aggregate positive surplus assumption (S).

\section{Failure of the First Fundamental Theorem OF WELFARE ECONOMICS}

Pareto-inefficiency is clear for any two-part tariff equilibrium concept that allows the hookup charge to exclude some households from purchasing the monopoly good, when these households are willing to buy the good at marginal cost, but receive insufficient surplus to justify paying the hookup charge. ${ }^{10}$ We avoid such inefficient equilibria by construction; our hookup charge is defined to be a fraction of consumer surplus.

Nonetheless, the examples of Fig. 1 and footnote 7 demonstrate that even though our constructed TPMCP equilibria avoid inefficient exclusion, they may be Pareto-inefficient. Hence there can be no general counterpart in this setting to the First Fundamental Theorem of Welfare Economics. The principal source of inefficiency is that with nonconvex technology, local optimal are not generally global optima. ${ }^{11}$

\section{The Second Fundamental Theorem of Welfare Economics}

Although a TPMCPE need not be Pareto-efficient, two-part marginal cost pricing can decentralize some Pareto-efficient allocations. Interestingly,

${ }^{10}$ This point is in Oi [16] for partial equilibrium.

"There is a positive efficiency result by Vohra [20] for the case two goods and one firm. This monopoly firm has a pure set-up cost technology, i.e., the only nonconvexity results from a set-up cost. He shows that there exist two-part marginal cost pricing equilibria which are efficient for this case. Some generalizations of this result in higher dimensions are to be found in Moriguchi [15]. 
some efficient allocations cannot be decentralized by a two-part marginal cost pricing equilibrium, as we show in the example below.

This example is also significant in that it shows that ordinary marginal cost pricing equilibria are not necessarily two-part tariff equilibria. In contrast, all efficient allocations can be supported by an ordinary marginal cost pricing equilibrium (see Bonnisseau and Cornet [4]).

EXAmPLE. Failure of Second Fundamental Theorem of Welfare Economics (Local Unwillingness Case). The basic intuition of the example is that marginal cost prices are guided by the local structure of production sets, while price-taking consumers are (mis)guided by linear extrapolation. Non-convexities break the connection between local conditions and the global search for the best allocations.

Consider the following economy. There are two goods, 0 and 1, produced by

$$
y^{0}=f^{0}\left(L^{0}\right)=\left\{\begin{array}{ll}
0 & \text { for } L^{0} \leqslant 1 / 10 \\
L^{0}-1 / 10 & \text { for } \pi / 2-1 / 10 \geqslant L^{0} \geqslant 1 / 10 \\
\pi / 2-2 / 10+ & \sin \left(L^{0}-(\pi / 2-1 / 10)\right) \\
& \text { for } \pi / 2 \geqslant L^{0} \geqslant \pi / 2-1 / 10
\end{array}\right\}
$$

and

$$
y^{1}=f^{1}\left(L^{1}\right)=\left\{\begin{array}{ll}
L^{1} & \text { for } \quad L^{1} \leqslant 1 / 3 \\
1 / 3+\sin \left(L^{1}-1 / 3\right) & \text { for } \pi / 2 \geqslant L^{1}>1 / 3
\end{array}\right\} .
$$

There is one household with labor endowment $\bar{L}=\pi / 2$ and utility function $U\left(x_{0}, x_{1}, L\right)=x_{0}+x_{1}$. The resource constraint is $L^{0}+L^{1}+L \leqslant \bar{L}$.

Efficient allocations are characterized by

$$
\left\{\begin{array}{l}
x_{0}=y_{0}=L^{0}-1 / 10=\pi / 2-L^{1}-1 / 10 \\
x_{1}=y_{1}=L^{1} \in[1 / 10,1 / 3]
\end{array}\right\} .
$$

In order to sustain an allocation of this form as an equilibrium we require a wage rate $w=p^{0}=p^{1}$. The required hookup is $q^{0}=\frac{1}{10} w$. But $q^{0}>0$ will not willingly be paid since the household's surplus from good 0 is nil. Hence the allocation cannot be sustained as an equilibrium. This emphatically is a case where the household is unwilling to finance the natural monopoly's losses.

Consider Fig. 2. GHCDJ is the production frontier. The segment GH represents the set-up cost (in terms of $x_{1}$ ) of producing $x_{0}$. BCDE is an indifference curve. The segment $\mathrm{CD}$ is the set of Pareto-efficient points. The line BCDE is the candidate budget constraint for decentralizing an 


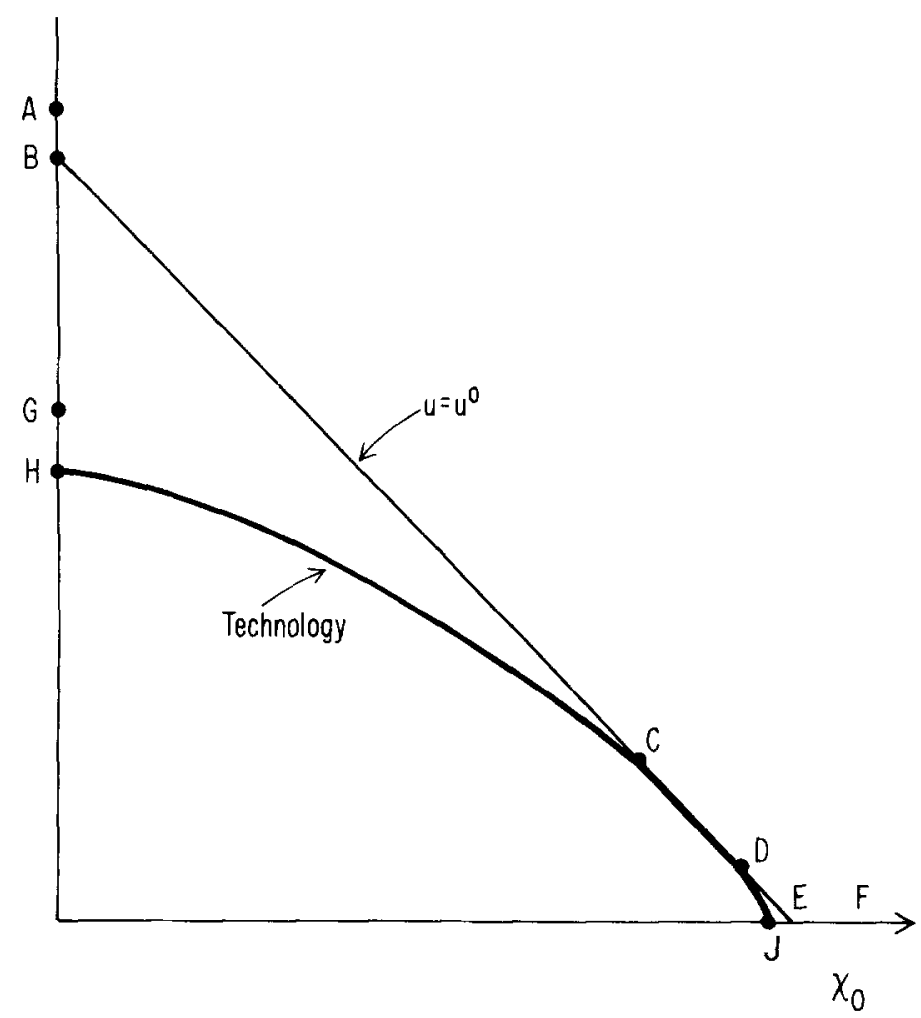

FIG. 2. Failure of the second fundamental theorem.

efficient allocation. It can also be regarded as the household budget frontier (evaluated at marginal cost price on $C D$ ) after subtractions of the imputed loss from firm 0 . The point $A$ represents the value of labor endowment in terms of $x_{1}$ ( $F$ does so in terms of $x_{0}$ ). Notice that there is coincidence of the budget line and the highest achievable indifference curve over their whole lengths. Therefore, the willingness to pay for access to good 0 is nil. Losses incurred by firm 0 are necessary to achieving an efficient allocation but will not willingly be paid by a price-taking consumer as a hookup charge for access to good 0 .

This example shows once again that some ordinary marginal cost pricing equilibria are not two-part marginal cost pricing equilibria; the second fundamental theorem of welfare economics holds for the former (see Bonnisseau and Cornet [4]).

Nevertheless, many Pareto-efficient allocations can be decentralized as two-part tariff equilibria. They key starting point is to define candidate supporting prices $\hat{p}$ for a given efficient allocation $\hat{a}$. One can then define 
income at that allocation and consumer surplus for each household. Theorem 2 then states that if at $\hat{p}$ the aggregate consumer surplus from the natural monopoly good covers the monopoly's losses, then the pair $\langle\hat{a}, \hat{p}\rangle$ is a two-part tariff equilibrium.

We need to recall two standard definitions before we can state Theorem 2. A compensated two-part marginal cost pricing equilibrium is defined the same as a TPMCPE above except that we replace utility maximization condition (a) of the definition. The condition that replaces (a) is: $\left(a^{\prime}\right)$ households minimize the expenditure needed to achieve the utility level. Following Arrow and Hahn [1] (p. 118), we say that household $h$ is indirectly resource-related to household $h^{\prime}$ if household $h^{\prime}$ desires the endowments of some third household $h^{\prime \prime}$, while $h^{\prime \prime}$ in turn desires the endowment of $h$.

THEOREM 2. Let $\left.\hat{a} \equiv\left\langle\hat{x}^{h}\right),\left(\hat{y}^{f}\right)\right\rangle$ be a Pareto-efficient allocation and choose an associated marginal cost price vector, $\hat{p} \in \varphi^{f}\left(\hat{y}^{f}\right)$. If at the prices $\hat{p}, \sum s^{h}\left(\hat{p},\left(\hat{y}^{f}\right)\right)>-\hat{p} \cdot \hat{y}^{0}$, then $\langle\hat{a}, \hat{p}\rangle$ is a compensated TPMCPE. If, in addition, every household is indirectly resource-related to every other household, then $\hat{a}$ is a full two-part marginal cost pricing equilibrium after suitable redistribution of income. ${ }^{12}$

Proof. Use the version of the second welfare theorem in Bonnisseau and Cornet's [4] article on valuation equilibrium and Pareto optimum to find the supporting marginal cost price vector $\hat{p}$. Then, as they point out in their Remark 2.1, $\hat{x}^{h}$ minimizes $p \cdot x^{h}$ over the "better than" set $\hat{P}^{h}=$ $\left\{x^{h} \in X^{h} \mid U^{h}\left(x^{h}\right) \geqslant U^{h}\left(\hat{x}^{h}\right)\right\}$ and $\hat{y}^{f}$ maximizes $\hat{p} \cdot y^{f}$ over $Y^{f}$ for $f \geqslant 1$. For $f=0, p \in \varphi^{0}\left(y^{0}\right)$. This is a compensated equilibrium.

We must find a set of hookup charges $\hat{q}^{h} \equiv q^{h}\left(\hat{p},\left(\hat{y}^{f}\right)\right)$ and incomes $\hat{r}^{h}$ such that: (1) for all $h$, $\hat{x}^{h}$ maximizes $U^{h}$ subject to $\hat{p} \cdot \hat{x}^{h}+\hat{q}^{h} \leqslant \hat{r}^{h}$, (2) $\sum \hat{q}^{h}=\max \left(-\hat{p} \cdot \hat{y}^{0}, 0\right)$ and (3) $\sum \hat{r}^{h}-\sum \hat{q}^{h}=\hat{p} \cdot\left(\sum_{f=0}^{F} \hat{y}^{f}+\omega\right)$.

By assumption, $\sum s^{h}>0$ so one can define

$$
\hat{\lambda}=\frac{\max \left(-\hat{p} \cdot \hat{y}^{0}, 0\right)}{\sum s^{h}\left(\hat{p},\left(\hat{y}^{\prime}\right)\right)} .
$$

Let $\hat{q}^{h} \equiv \hat{\lambda} s^{h}\left(\hat{p},\left(\hat{y}^{f}\right)\right)$. Thus condition (2) is satisfied.

Define $\hat{r}^{h}=\hat{p} \cdot \hat{x}^{h}+\hat{q}^{h}$. Then $\sum_{h}\left(\hat{r}^{h}-\hat{q}^{h}\right)=\sum_{h} \hat{p} \cdot \hat{x}^{h}$. Now, Paretoefficiency of $\hat{a}$ and local non-satiation guarantees that $\sum \hat{x}^{h}=\sum \hat{y}^{f}+\omega$. Thus, $\hat{p} \cdot \sum \hat{x}^{h}=\hat{p} \cdot\left(\sum \hat{y}^{f}+\omega\right)$, and so $\sum_{h}\left(\hat{r}^{h}-\hat{q}^{h}\right)=\hat{p} \cdot\left(\sum \hat{y}_{f}+\omega\right)$. Condition (3) is met.

\footnotetext{
${ }^{12}$ Duncan Foley provided inspiration for this result.
} 
To show that $\hat{x}^{h}$ actually maximizes $U^{h}$ subject to $\hat{p} \cdot x^{h}+\hat{q}^{h}+\hat{q}^{h} \leqslant \hat{r}^{h}$ requires showing that $\hat{r}^{h}-\hat{q}^{h}>\inf \hat{p} \cdot X^{h}$. But this is implied by indirect resource relatedness (cf. Arrow and Hahn [1]).

\section{ConClusions}

Nonlinear pricing has been explored in a general equilibrium model with increasing returns to scale. We considered a private ownership enconomy with a regulated natural monopoly. Competitive firms with nonincreasing returns price at marginal cost. By contrast, the natural monopoly uses a two-part tariff: set price equal to marginal cost, and set a hookup charge for access rights to the monopoly good so as to recover the losses.

Two-part marginal cost pricing equilibria are not generally Paretoefficient. This is in contrast to the impression left by much of the partial equilibrium literature on two-part tariffs. Further, we show by example that it is not always possible to support an efficient allocation as a two-part tariff equilibrium. Hence, both the First and Second Fundamental Theorems of Welfare Economics may fail.

Despite non-convexities in both producer and consumer possibility sets, the use of approximate equilibrium is unnecessary. For this purpose, we introduce a notion of consumer surplus as the willingness to pay for access to the increasing returns good. We assume that aggregate surplus (the sum of individual consumer surpluses) exceeds the losses of the regulated monopoly. The individual's hookup charge is set to fixed fraction of his consumer surplus. Then exact two-part marginal cost pricing equilibria exist; they require positive production of the increasing returns good. Further, under the assumption on aggregate surplus, a counterpart to the Second Fundamental Theorem of Welfare Economics for two-art tariffs has been established.

The source of the market failure here is not attributable to missing markets. The market failure in the case of increasing returns comes from the fact that the first order necessary conditions are not sufficient for an optimum, absent convexity. The myopic nature of marginal cost pricing makes it an unreliable guide to global optimality with non-convexities. Hence, merely instructing the regulated monopoly to charge marginal cost prices and nonexclusionary hookups is not sufficient to attain efficiency. Fortunately, a Pareto-efficient allocation is supportable as two-part marginal cost pricing equilibria whenever that efficient allocation has aggregate positive surplus.

The informational requirements for exact calculations of consumer surplus are extreme. But it is not implausible that willingness to pay is 
correlated with income. In some parts of the country, the electric utility charges a lower ("lifeline") hookup for households that can pass a means test.

\section{REFERENCES}

1. K. J. Arrow and F. Hahn, "General Competitive Analysis," Holden-Day, San Francisco, 1971.

2. P. Beato and A. Mas-Colflel, On marginal cost pricing with given tax-subsidy rules, J. Econ. Theory 37 (1985), 356-365.

3. J. M. Bonnisseau AND B. CoRnet, Equilibria and bounded loss pricing rules, J. Math. Econ. Symposium (1988a), 119-147.

4. J. M. Bonnisseau and B. Cornet, Valuation equilibrium and Pareto optimum in nonconvex economies, J. Math. Econ. Symposium (1988b), 293-308.

5. KIM Border, "Fixed Point Theorems with Applications to Economics and Game Theory," Cambridge Univ. Press, New York, 1985.

6. D. J. Brown and G. M. Heal, Equity, efficiency, and increasing returns. Rev. Econ. Stud. 46 (1979), 571-585.

7. D. J. Brown, G. M. Heal, M. A. Khan, and R. Vohra, On a general existence theorem for marginal cost pricint equilibria, J. Econ. Theory 38 (1986), 371-379.

8. R. COASE, The marginal cost controversy, Economica 13 (1946), 169-189.

8. B. Connet, General equilibrium theory and increasing returns: Presentation, J. Math. Econ. Symposium (1988), 103-118.

10. G. Debreu, "Theory of Value," Wiley, New York, 1959.

11. R. GUESNERIE, Pareto-optimality in non-convex economies, Econometrica 43 (1975), 1-29.

12. W. P. Heller and R. M. Starr, Equilibrium with non-convex transaction costs, monetary and non-monetary economies, Rev. Econ. Stud. 43 (1976), 195-215.

13. K. KamiYa, Existence and uniqueness of equilibria with increasing returns, J. Math. Econ. Symposium (1988).

14. W. A. Lewis, The two-part tariff and The two-part tariff: A reply, Econometrica 7 (1941), 249-270 and 399-408.

15. C. Moriguchi, Two part marginal cost pricing in a general equilibrium model, Department of Economics, Osaka, 1991.

16. W. Y. O1, A Disneyland dilemma: Two-part tariffs for a mickey mouse monopoly, Quart. J. Econ. 85 (1971), No. 1.

17. Martine Quinzil, "Increasing Returns and Efficiency," Cambridge Univ. Press, Cambridge, in press.

18. N. RugGles, Recent developments in the theory of marginal cost pricing, Rev. Econ. Stud. $17(1949,1950), 107-126$.

19. Symposium on General Equilibrium and Increasing Returns, J. Math. Econ. 17 (1988), Nos. 2/3.

20. R. Vohra, On the inefficiency of two-part tariffs, Rev. Econ. Stud. 57, No. 3 (1990), 415-438. 